Meta

Journal des traducteurs

Translators' Journal

\title{
Terminologie : l'ombre du concept
}

\section{François Gaudin}

Volume 41, numéro 4, décembre 1996

\section{La dénomination}

URI : https://id.erudit.org/iderudit/002813ar

DOI : https://doi.org/10.7202/002813ar

Aller au sommaire du numéro

\section{Éditeur(s)}

Les Presses de l'Université de Montréal

\section{ISSN}

0026-0452 (imprimé)

1492-1421 (numérique)

Découvrir la revue

\section{Citer cet article}

Gaudin, F. (1996). Terminologie : l'ombre du concept. Meta, 41(4), 604-621. https://doi.org/10.7202/002813ar

\section{Résumé de l'article}

La délimitation de la terminologie comme discipline se trouve souvent justifiée, directement ou indirectement, par le recours à la notion mal délimitée de concept. L'examen des idées de quelques philosophes du langage contemporains permet de mieux comprendre la place des concepts face aux pratiques langagières et à l'activité de catégorisation. La conceptualisation étant inséparable de la mise en mots, il en résulte que la construction textuelle des concepts peut être étudiée en tant qu'activité discursive. D'où il découle la pos-siblité d'articuler la description des termes en recourant à l'opposition entre signifié et concept, opposition qui permet de mieux régler la description de la polysémie. 


\title{
TERMINOLOGIE: L'OMBRE DU CONCEPT $^{1}$
}

Francols Gaudin

URA CNRS 1164, Université de Rouen, Rouen, France

\begin{abstract}
Résumé
La délimitation de la terminologie comme discipline se trouve souvent justifiée, directement ou indirectement, par le recours à la notion mal délimitée de concept. L'examen des idées de quelques philosophes du langage contemporains permet de mieux comprendre la place des concepts face aux pratiques langagières et à l'activité de catégorisation. La conceptualisation étant inséparable de la mise en mots, il en résulte que la construction textuelle des concepts peut être étudiée en tant qu'activité discursive. D'où il découle la possiblité d'articuler la description des termes en recourant à l'opposition entre signifié et concept, opposition qui permet de mieux régler la description de la polysémie.
\end{abstract}

\begin{abstract}
The notion of concept is often used to justify the status of terminology as an independent discipline and yet "concept" is not a particularly well defined notion. The works of some contemporary philosophers specializing in language enable us to gain a better insight into the part played by concepts in language and categorization. Conceptualization cannot be separated from speech activity and so concept construction can be studied as a discursive activity. Thus, terms can be described thanks to the opposition between signified and concept, an opposition which makes it possible to improve the description of polysemy.
\end{abstract}

La tante Irma n'était la tante de personne. On l'appelait la tante Irma parce que, n'ayant pas de neveux, elle semblait plus propre qu'une autre à représenter le concept tante à l'état pur, en dehors de toute contingence. (Alexandre Vialatte, Le fluide rouge, éd. Le dilettante, 1990, p. 17.)

\section{LE CONCEPT, UNE NOTION CENTRALE EN TERMINOLOGIE}

La délimitation de la terminologie comme discipline se trouve souvent justifiée, directement ou indirectement, par le recours au concept (ou à la notion). (Sur ce point, Rey (1979: 17 et suiv.).) Et l'on rencontre fréquemment le parallèle entre signes (unions de signifiants et de signifiés) et termes (unions de noms et de concepts), usage qu'entérine la norme ISO 1087. Cependant, si un tel parallélisme peut séduire, son examen soulève bien des questions. Notamment, le fait que le nom ne saurait se réduire à un seul signifiant: que deviendrait alors le signifié du terme? De plus, cette conception présente l'inconvénient de réduire les terminologies à des nomenclatures.

\subsection{LES NOMENCLATURES : UN CAS PARTICULIER}

Certes, les nomenclatures existent et sont utiles, mais l'anomalie qu'elles constituent au sein du lexique ne saurait masquer le fonctionnement général des vocabulaires servant dans les situations de transmission de connaissances et de travail, pour définir un peu largement l'éventail des pratiques langagières concernées. 
Les nomenclatures existent et elles permettent d'étiqueter de façon univoque des réalités très bien délimitées: aciers, composés chimiques, modèles d'automobiles, etc. Cependant, il convient ici de rappeler la position de Ferdinand de Saussure sur cette question. À plusieurs reprises, l'auteur du Cours de linguistique générale s'attaque à toute réduction de la langue à une nomenclature.

Il y a d'abord la conception superficielle du grand public: il ne voit dans la langue qu'une nomenclature, ce qui supprime toute recherche sur sa nature véritable. (1985:34)

Pour certaines personnes, la langue, ramenée à son principe essentiel, est une nomenclature, c'est-à-dire une liste de termes correspondant à autant de choses. [...] Cette conception est critiquable à bien des égards. Elle suppose des idées toutes faites préexistant aux mots [...] Enfin, elle laisse supposer que le lien qui unit un nom à une chose est une opération toute simple, ce qui est bien loin d'être vrai. (1985:97)

Sur ce point, la critique de Saussure est fondamentale pour notre propos.

Il en découle que le fonctionnement général et normal des langues oblige à tenir pour essentielle la distinction entre signification et désignation, distinction que les nomenclatures permettent d'écraser volontairement; au lieu d'impliciter un secteur de l'expérience, le signe vise alors un référent particulier. Par exemple, dans la nomenclature chimique, méthyle $\mathrm{CH} 3$ sert à désigner un corps caractérisé et ne possède guère de sens hors cette nomenclature. Mais ce fonctionnement particulier, volontaire et concerté qui caractérise les nomenclatures ne se comprend qu'au regard du système linguistique. Et, sur ce problème, Tullio de Mauro semble prolonger avec justesse les conceptions de Saussure.

Ce n'est pas l'objet qui est la base du sens des mots, mais c'est au contraire l'usage du mot qui rassemble des expériences disparates du point de vue perceptif, constituant ainsi, dans des conditions et pour des raisons socialement déterminées, ce que l'on appelle l'objet. (1985: 439)

Il est donc possible de refuser dans le même temps la confusion entre sens et référence (que l'on excuse plus facilement chez Johnson au XVIII ${ }^{e}$ siècle), qui réduit le signe à un nom de chose, et le conceptualisme, qui réduit le sens de certaines unités lexicales à un contenu cognitif organisé. C'est là ce à quoi tendent toutes les conceptologies, depuis Leibniz au moins, et certaines approches de la sémantique, ce dont témoigne avec humour Hilary Putnam:

La notion de sens est une catastrophe si elle est conçue comme quelque chose entre les signes et le monde. J'ai parfois comparé cette notion aux deux faces du Velcro, avec une face qui serait cousue au cerveau, tandis que l'autre serait cousue aux choses... et il y aurait sens parce que ça colle. (1992: 55)

Il suffit, pour aborder ces questions, de rester assez près de la pensée saussurienne et de distinguer à des fins heuristiques, au moins provisoirement, le concept et le signifié. D'ailleurs, il suffit de remarquer que dès lors qu'une unité lexicale est utilisée hors nomenclature avec des valeurs nouvelles, son signifié se distingue du concept délimité.

\subsection{SIGNIFIÉ / CONCEPT: UNE DISTINCTION OPÉRATOIRE?}

Prenons un exemple simple catalyser correspond, dans la bouche d'un chimiste, à un concept opératoire bien délimité. Mais ce terme, apparu au milieu du XIX ${ }^{e}$, a été pris dans des discours assez nombreux pour acquérir une dimension culturelle qui l'a rendu susceptible, dans les années 50, d'emplois métaphoriques: un décision peut catalyser le mécontentement. Plus récemment, le terme clone s'est diffusé rapidement dans son sens 
premier et a vite suscité des usages métaphoriques; on parle ainsi d'un «clone» d'IBM; les exemples sont légion...

\subsection{CONCEPT OU NOTION?}

Si l'on accepte ce qui précède, il importe donc de distinguer entre signifiés et concepts. Et pour ce faire, il faut se demander ce que l'on peut entendre par concept et à quelles réalités pratiques ce terme permet de renvoyer.

Certes, lorsque l'on parle de concept, le danger de sombrer dans la métaphysique est réel. Toutefois, si l'on tient à dépasser la description phénoménale du concret et à sortir de l'empirisme, il importe de chercher à cerner les difficultés théoriques que soulève l'utilisation, centrale en terminologie, de cette notion.

Mais parlerons-nous de concept ou de notion?

On connaît la portée et la validité des décisions de normalisation. Sur de telles questions, elles peuvent rendre service, mais on ne saurait en tirer quelque enseignement. Lorsque l'ISO introduit une relation de synonymie entre les deux termes (norme ISO 1087, 1990), elle offre une caution pour simplifier la réflexion. C'est cette voie que nous suivrons ici, mais il convient, avant d'aller plus loin, de souligner le fait que la distinction entre les deux termes est opératoire. Ainsi, pour Michelle Goupil, historienne des sciences,

[...] la notion préfigurant le concept prend sa source, ou bien dans des sensations physiologiques des phénomènes physicochimiques, ou bien dans des conceptions anthropomorphiques tentant d'expliquer les phénomènes observés. (1988:25)

Dans cet esprit, travail, énergie ou force constituent tout d'abord des notions, pouvant, malgré leur enracinement dans une expérience sensible, accéder au statut de concepts au prix d'une opération abstractisante, qui peut prendre la forme d'une formalisation. L'auteur propose un critère précis, la capacité à être représentée par une grandeur mesurable et mathématiquement formulable. Cette opposition témoigne de la différence de distributions que connaissent les deux termes; en effet, le mot notion connait une plus grande diversité d'emplois et peut renvoyer à une connaissance empirique, intuitive, fragmentaire ou modeste.

Suivant partiellement cette opposition, nous parlerons préférentiellement de concept, puisque nous nous intéresserons principalement à des contenus de connaissances sachant que de nombreux auteurs utilisent les deux termes comme des para-synonymes.

\section{LE CONCEPT: QUELQUES PROBLÈMES THÉORIQUES}

Il paraît difficile d'évoquer le problème du concept sans jeter un œil, même profane, sur le statut que lui reconnaissent les philosophes; après tout, nous n'userions pas de l'acception moderne sans Descartes et Kant. De plus, la problématique des terminologues entretient, depuis sa fondation, d'étroites relations avec celle des philosophes du langage (sur ce point, voir Slodzian 1993).

\subsection{UNE CONSTRUCTION DU MONDE}

Nous ne ferons pas ici l'archéologie de la discipline, mais nous nous contenterons de souligner quelques caractéristiques de réflexions menées sur le concept pouvant intéresser notre propos. Sur des points aussi riches de littérature et difficiles, nous serons, fatalement, lacunaire.

Dans son approche de la conceptualisation, Ernst Cassirer oppose deux approches théoriques. Dans la première, le concept est une abstraction fondée sur des regroupements 
d'objets possédant des caractères communs; il est alors extrait de la réalité. Une telle conception se retrouve dans la norme ISO 1087 :

notion: unité de pensée constituée par abstraction à partir des propriétés communes à un ensemble d'objets.

La théorie opposée, et que défend Ernst Cassirer, envisage la conceptualisation comme fondée sur un acte. Acte perceptif pour les concepts empiriques; acte intellectuel pour les concepts de type mathématique relevant de «la libre production de certains enchaînements rationnels» $(1977: 23)$.

\subsection{DE L'ACTE INTELLECTUEL À L'OUTIL TEXTUEL}

Cet acte, fondateur de la construction intellectuelle, se trouve au centre d'une vision dans laquelle il s'agit moins de restituer le monde des choses et des représentations sensibles que de le redessiner en lui substituant un ordre d'un autre type. L'abstraction doit alors être comprise comme «l'accomplissement, dans l'intuition vivante, des actes mentaux saisis dans leur riche diversité comme dans leur autonomie» (Cassirer 1977: 38). Et le lien entre le concept et sa dénomination est tel que toute reformulation d'un concept, événement textuel, entraîne en même temps un renouvellement de l'ensemble du champ qu'il contrôle et ordonne.

L'acte permet la construction dynamique de concepts qui répugnent à engendrer de simples décalques. Leur existence est alors toute conventionnelle, dans la mesure où il faut reconnaître, au fondement de toute abstraction, un acte d'identification, identification qui permet d'insister sur le rôle de l'énonciateur, auteur de l'acte de naissance du concept.

Sur ce point, les travaux sociolinguistiques ont démontré le rôle crucial des énonciateurs en matière de dénomination (Mortureux 1984). Or, pour autant que la connaissance a partie liée avec le langage, la dénomination d'un concept doit posséder des traits communs avec le fonctionnement général du processus de dénomination. Et Cassirer affirme clairement le rôle du langage dans la construction des concepts, même les plus formalisés :

[...] si haut que le concept pur puisse s'élever au-dessus du monde des sens dans l'empire de l'idéel et de l'«intelligible», il n'en retourne pas moins toujours en dernier ressort, d'une façon ou d'une autre, à cet organe «mondain et terrestre» qu'il possède dans le langage. (1972:364)

Dans ce travail de pensée par lequel l'esprit construit ses représentations, le mot ne reçoit son sens que de la proposition prédicative et de l'enchaînement du discours; mais cela pose la question de la construction, à partir des matériaux sémiotiques du langage, du concept. Comment un concept se construit-il?

2.2.1. LA CONSTRUCTION DU CONCEPT

Plusieurs travaux, menés dans des orientations différentes, mais toujours en partant des textes, donc des signes qui sont les seuls observables, permettent de défaire un peu l'écheveau de cette délicate question.

Selon Frédéric Cossutta, la construction du concept procède d'un «procès d'instauration du sens» (1989:41). Cet ensemble de procédures, qui relèvent d'une construction terminologique par un auteur donné, est lié à une sémantisation conceptuelle, qui résulte d'une construction textuelle. Nous retrouvons la prise en compte, par Cassirer de la dimension discursive.

Dans cette optique, il n'y a pas construction du concept par un acte isolé, de type définitoire; au contraire, on ne saurait dissocier un concept de l'ensemble des usages qui en sont faits. De ce point de vue, le nom du concept est solidaire d'autres signes, ses 
cooccurrents, qui participent à la verbalisation du contenu conceptuel : que serait le concept géométrique de «point», si sa dénomination ne se rencontrait régulièrement associée à droite, nom d'un concept qui lui est très proche? Et si la définition se voit conférer un rôle propre, c'est celui de proposer des règles de réécriture qui permettront le fonctionnement aisé de la synonymie et de la paraphrase.

La procédure définitoire peut également être rendue nécessaire pour des raisons proprement linguistiques, du fait de la polysémie de la dénomination. C'est ce qui advient lorsqu'il s'agit d'arracher des termes familiers à des acceptions usuelles pour opérer une réassignation sémantique : on est alors dans un réglage du sens, volontaire quand on fait consciemment cet effort de réassignation sémantique. Encore y aurait-il lieu ici de distinguer les cas où la dénomination joue, ou non, sur le sens initial. En effet, la pure homonymie reliant moment, terme de physicien, à moment, du temps quotidien, s'oppose à la diversité des conceptualisations attachées au terme récepteur dans divers secteurs du savoir. Dans ce dernier cas de figure, le réglage du sens dans l'interaction joue, sans décision préalable, sur les virtualités des programmes de sens implicités sous le terme récepteur.

\subsubsection{UNE CONSTRUCTION À VALIDER}

Il importe donc de tenir compte des conditions d'énonciation du concept. Cela nous conduit à mettre en avant ce qui fonde son caractère social : il n'est de concept que validé, et cette validation consiste en ce que sa réception par des interlocuteurs soit attestée. Le concept doit être validé socialement, au sens où tout homme de pensée est un homme public.

Comme le dit Karl Otto Apel :

[...] la validité de la pensée solitaire est principiellement dépendante de la justification des énoncés linguistiques dans la communauté effective d'argumentation. Il n'est pas possible pour un seul être de suivre une règle et de valider sa pensée dans le cadre d'un «langage privé». La pensée est bien plutôt publique par principe. (1987: 90)

On retrouve une idée comparable chez Hilary Putnam, dans les critiques qu'il adresse aux mentalistes, lorsqu'il insiste sur le fait que la signification «ne dépend pas simplement de ce qui est dans notre tête, mais aussi de ce qui est dans notre environnement et de la manière dont nous interagissons avec cet environnement» (1990: 47).

Nous pouvons conclure de ce rapide parcours que le concept est une construction, résultant d'un acte d'identification, volontaire, par l'instauration du sens, et validée, puisque de caractère public. Cette construction participe à un univers de connaissance et sa dénomination constitue un acte fondateur, pour autant qu'elle lie le concept à un ensemble d'usages linguistiques qui réorganisent (par la sémantisation conceptuelle) un pan du monde construit, ce que les linguistes appellent, nous le verrons, la logosphère.

Mais nous en sommes resté pour l'instant aux procédures de construction des concepts sans nous interroger sur leur contenu même. La solution traditionnelle consiste à réduire le sens d'un concept à sa définition et, particulièrement en terminologie, à «le dériver à partir d'autres concepts (objets) en remontant par degré jusqu'aux concepts (objets) fondamentaux» (Slodzian 1993: 227). Cette perspective est difficilement compatible avec le primat accordé aux textes qui découle de ce qui précède. Or ce problème se pose en pratique dans la confection de dictionnaires de... concepts : comment faut-il les décrire?

\subsection{LES NOTIONS SUR LES NOTIONS}

C'est là un des problèmes que s'est posé Sylvain Auroux en tant que lexicographe de la philosophie lorsqu'il a dirigé le tome II de l'Encyclopédie philosophique 
universelle. Le domaine est en effet privilégié pour notre réflexion dans la mesure où la philosophie est toujours affaire de textes, de discours dans lesquels les unités linguistiques se contextualisent et finissent par acquérir des usages spécifiques, variables en fonction des choix des auteurs. L'ouvrage s'intitule «Les notions philosophiques», mais l'usage de l'auteur fait de notion et de concept d'occasionnels synonymes (1990: XI).

Cette expérience lexicographique mérite qu'on s'y attarde, dans la mesure où les auteurs ont rencontré des difficultés pour parvenir à concevoir clairement, et sans contredire les acquis contemporains des sciences du langage, le rapport entre la valeur linguistique des formes lexicales et les notions, unités plus vastes. Pour ce faire, ils ont été conduits à poser que «l'ensemble des notions sur quelque chose est la notion concernant cette chose» (1990: XI). Derrière l'apparent paradoxe, il faut lire l'affirmation que les notions sont des constructions discursives ou textuelles.

Ici encore, les textes priment. Et seule la prise en compte des discours permet de comprendre comment se construisent les notions, de mettre en lumière les procédés utilisés et de souligner combien ces notions, loin d'appartenir à un monde intangible, sont sans cesse reprises, discutées, reformulées, même si certaines d'entre elles, de par l'existence de cohérences conceptuelles ou de facteurs institutionnels, ont acquis une stabilité relative. Il s'agit de toute façon d'une autre évidence que celle des objets du monde sensible. Ce qu'il faut considérer comme des notions, ce sont, non pas des réalités données, mais des développements, des discours, des constructions.

Cette façon d'envisager les notions permet non seulement de replacer au premier plan les textes qui leur donnent sens, mais aussi de mettre en lumière l'importance de leur histoire. C'est là un thème bien étudié en histoire des sciences, ou épistémologie; l'histoire des concepts participe de leur compréhension. Le phénomène est peut-être plus patent à leur sujet, mais ce n'est pas là un privilège des seules notions philosophiques.

Ce travail lexicographique est intéressant à un autre titre, c'est qu'il souligne le fait que les notions philosophiques peuvent être liées à la valeur linguistique des mots qui permettent de les dénommer. C'est ainsi que l'auteur précise que la valeur lexicale d'un mot peut se confondre avec la notion à laquelle ce mot renvoie. C'est le cas lorsque le mot est simplement un nom de notion. On retrouve là l'affirmation du rapport d'inclusion entre la langue générale et ses sous-ensembles, particulièrement ceux employés par des communautés socio-professionnelles.

C'est donc l'ensemble du fonctionnement socio-linguistique, socio-discursif et historique des termes qui doit servir de point de départ. Les concepts doivent être abordés en incluant leur genèse dans des textes et en prenant en compte leur enracinement dans une histoire et un patrimoine linguistique. Mais peut-être n'avons-nous pas suffisamment l'habitude de penser aux significations comme à des entités historiques?

C'est en ce sens qu'Alain Rey considère que c'est grâce à la terminologie, discipline «dont l'exercice effectif est noyé dans les activités documentaires et traductrices», que peut se pratiquer «l'archéologie des constructions conceptuelles, des systèmes idéologiques et scientifiques, ou en un terme trop simple l'histoire des idées» (1990: 778). Faute de quoi, on ne travaille que sur des modèles, des réductions, bref des représentations du réel qui oublient le caractère dynamique et historique des concepts (sur ce point, voir Boisson dans ce volume).

\subsection{LA LOGOSPHÈRE}

Une approche plus strictement linguistique de ces questions est possible en recourant à la notion de logosphère, proposée par le père de la praxématique, Robert Lafont, dans ses réflexions sur les liens entre le langage et la réalité : «la logosphère, c'est le maillage du sens enveloppant le réel» (1993: 292). 
Comme nous l'avons montré ailleurs (Gaudin 1993b), cette notion permet de résoudre certaines difficultés que peuvent poser les rapports de la langue dénommante et du monde dénommé. À notre sens, elle permet de répondre à deux sortes d'interrogations: d'une part, celles que soulève la perpétuelle tension qui oppose le mouvement créatif de l'individualité de la parole et la fixité des normes, linguistiques et culturelles, et, d'autre part, celles que posent les concurrences de référence et la mutabilité des valeurs de vérité.

Prenons en exemple le problème des changements de théories de référence. Selon Thomas Kuhn, «bien que le monde ne change pas après un changement de paradigme, l'homme de science travaille désormais dans un monde différent» (1972: 148). Et cette contradiction apparente l'étonne et le conduit à souhaiter que la science puisse exprimer ses analyses dans les termes d'un langage d'observation neutre. On retrouve là le vieux rêve leibnizien d'un langage scientifique universel et indépendant des langues particulières, en fait d'un pur métalangage.

Or la contradiction se trouve résolue si l'on considère que l'homme de science, comme tout locuteur, ne vit pas dans le monde brut, mais dans un monde mis en mots qui évolue et que le scientifique, au quotidien, contribue à édifier. Ce monde mis en mots, la praxématique le dénomme logosphère. Puisque la logosphère est construction, le dilemme de Kuhn disparaît: après un changement de théorie, l'homme de science ne travaille pas dans un monde différent, ni sur des choses différentes, mais le monde de langage qu'il construit, le sens qu'il donne aux choses et qui accompagne son action ont simplement changé. Les progrès de la connaissance nécessitent ainsi une perpétuelle réorganisation du monde construit auquel nous nous référons.

Les concepts sont aussi partie prenante de la logosphère. Leur sens est construit, maintenu, négocié dans les pratiques langagières. Un appareil formel peut faciliter leur fixation (pensons aux sciences exactes), mais n'oublions pas que même les formules et les théories de référence changent. La volonté de monosémie qui caractérise le fonctionnement social des termes ne doit pas occulter les tensions qui traversent des vocabulaires socialement situés et, comme tels, parcourus d'enjeux, mais aussi porteurs d'histoire. Loin de conduire à poser d'emblée une conceptologie transcendante, l'examen attentif de ces questions permet de pousser assez loin les modèles issus des linguistiques de l'interaction et l'on peut poser que la notion de négociation, bien travaillée dans les pratiques langagières, joue également un rôle déterminant dans l'activité de conceptualisation; c'est en ce sens que nous avons proposé de parler de «négociation cognitive» (Gaudin 1993d : 201). Mais c'est là un autre débat...

\section{DU CÔTÉ DE LA RÉFÉRENCE}

Ce qui précède nous a permis de mettre en avant l'importance d'une approche discursive et (socio-)linguistique pour cerner ce que l'on désigne par notion ou concept en terminologie. Mais il importe de chercher à mieux comprendre comment se fixent les normes permettant l'intercompréhension, normes qui fondent la terminologisation de vocables. Ces questions, notamment celles relatives à la référence, sont très travaillées dans le champ de la philosophie analytique. À défaut de pouvoir embrasser un large domaine qui ne relève pas de notre compétence, nous nous limiterons ici aux points de vue développés par Saül Kripke (1982) et par Hilary Putnam (1984, 1990, 1992).

Abordant les questions de la nomination, Saül Kripke insiste sur le caractère social de l'emploi des noms propres et des termes, qui ne permettent de faire référence que :

[...] grâce à notre interaction avec les autres locuteurs de la communauté, interaction en vertu de laquelle nous sommes liés au référent lui-même. $(1982: 82)$ 
Bien que raisonnant dans une perspective logicienne, Kripke trouve abusive la réduction d'objets concrets à une définition de type exacte; il prend pour exemples celles de l'or à son nombre atomique, de l'eau à $\mathrm{H}_{2} \mathrm{O}$, ou de la lumière à un flux de protons. Selon lui, il est préférable de substituer

[...] à l'idée de propriétés nécessaires et suffisantes, l'idée d'un faisceau de propriétés, dont seulement quelques-unes doivent être satisfaites dans chaque cas particulier. (1982:116)

Et l'on retrouve ici les questions posées en termes de prototype, notamment par les linguistes qui ont emprunté cette notion aux psychologues, et en termes d'«air de famille», pour reprendre la notion proposée par Wittgenstein $(1961: 147)$.

Concernant la référence, elle est initialement fixée par l'acte dénominatif, puis se transmet de maillon en maillon, de la même façon que pour le nom propre. Et dans le chaînage des interactions successives, des modifications peuvent prendre place qui - permettent d'expliquer le maintien des dénominations face à la mutation des référents.

Hilary Putnam adopte des positions analogues à celles de Kripke à propos de la référence, qu'il renvoie à un consensus social élargi à ce qu'il appelle «la contribution de l'environnement» (1990:68). On se trouve ici au cœur de la conception du philosophe des relations entre référence et signification (cette dernière étant interactionnelle).

Dans le cas d'objets naturels, la référence peut se trouver fixée de façon consensuelle par des normes; on détermine ainsi, par exemple, que certains objets sont des échantillons paradigmatiques, mais provisoires et révisables. Et dans les cas douteux, tout un chacun peut consulter des experts. En posant que la référence est un phénomène social, Putnam n'oublie pas que l'activité de langage, pour coopérative qu'elle soit, connaît une répartition des rôles: «il y a une division linguistique du travail».

\subsection{LES SIGNIFICATIONS NE SONT PAS DANS LA TÊTE}

Cette vision de la construction, socialement partagée et répartie, de normes linguistiques s'articule à une critique radicale du mentalisme dont le but est «d'identifier la signification d'un mot avec quelque chose qui se trouve dans l'esprit / cerveau de tout locuteur sachant utiliser le mot» (1990:55).

Pour lui, les significations ne sont pas dans la tête. Et ceci se retrouve dans sa conception du concept, qui relève de l'ordre individuel, mais sur le mode de l'agir. En effet, c'est ce critère qui lui permet d'opposer concept et image mentale, puisqu'«une image mentale qui n'est pas accompagnée de la capacité d'agir n'est qu'une simple image» (1984: 30).

La place qu'il accorde à l'action le conduit à lier l'existence de catégories conceptuelles à une mise en spectacle du monde, qui n'est pas éloignée de la construction de la logosphère dont nous parlions plus haut. Et si les objets peuvent paraître figurer sous des étiquettes, ce n'est qu'en vertu du fait que «ces étiquettes sont les outils que nous avons utilisés au départ pour construire une version du monde contenant ces objets» (Putnam 1984: 66). En se gardant ainsi de la conception de la langue comme nomenclature, on relativise l'importance des secteurs dans lesquels des «effets de nomenclature» sont réussis, grâce à une standardisation acceptée et à la stabilité des connaissances ou du monde construit.

En soulignant que les signes ne correspondent pas intrinsèquement à des objets, on restitue au langage toute la place qu'il occupe dans la conquête du savoir. Car, si ce savoir reçoit effectivement de l'extérieur, ou plutôt de l'expérience, des inputs, il convient de refuser que ceux-ci : 
[...] ne soient pas dans une certaine mesure influencés par nos concepts, par le vocabulaire que nous utilisons pour les rapporter et les décrire, ou qu'il y ait des inputs qui admettent une description unique, indépendante de tout choix conceptuel. (Putnam 1984 : 66)

\subsection{SENS : INCOMMENSURABILITÉ ET HOLISME}

C'est détruire là le vieux rêve de langue scientifique, universelle ou objective, mais on sait que «le caractère de 'métalangage' que revêt, pour certains, toute terminologie et tout code formel - selon le grand projet leibnizien - est partiellement fictif, à la fois limité et indéfini, chaque terminologie suscitant son propre métalangage» (Rey 1990: 776).

Poussée à l'extrême dans sa logique, la perception de ce hiatus - qui selon nous découle d'un oubli de la nature de construction linguistique du savoir — conduit à la thèse de l'incommensurabilité. Les notions scientifiques de «température» telles qu'utilisées par les scientifiques du XVII siècle et ceux d'aujourd'hui renverraient à des paradigmes distincts. En conséquence de quoi, elles seraient intraduisibles.

Pour Hilary Putnam, la solution de ce problème réside dans ce qu'il appelle joliment la «charité dans l'interprétation» (1984: 135). Il s'agit en quelque sorte de réserver une place au découpage proprement linguistique, auquel il est fait crédit, en deçà de la construction conceptuelle. Mais il s'agit là d'une interprétation de linguiste; que dit le texte?

Reprenons les grands traits de l'argumentation. Des locuteurs différents ont des prototypes perceptuels différents de l'herbe; leurs conceptions diffèrent selon l'époque (pas de photosynthèse dans la conception d'il y a deux siècles), mais les concepts qu'ils ont construits sont suffisamment communs pour qu'ils sachent que l'herbe est une plante. C'est ici qu'intervient le principe de «charité dans l'interprétation», qui nous fait tenir les deux acceptions pour équivalentes, et sans lequel on ne pourrait rien dire du tout de ce que désignaient ces mots il y a deux cents ans. En fait, sans prendre des exemples historiquement éloignés ou faire référence à des théories divergentes, toute interprétation repose sur cette «charité», dans la mesure où l'intercompréhension nécessite toujours de laisser de côté, quand nous interprétons, certaines différences de croyance. s'agit-il?

Cette position conduit à poser le principe du «holisme» de la signification. De quoi

En réaction au positivisme logique, dont on sait l'influence sur la terminologie, le holisme de la signification s'oppose au principe qui veut que la signification des mots soit expliquée ou fixée par une définition. La croyance qui fonde cette attitude réside dans le fait que la plupart des termes ne peuvent pas être définis de façon fixe, la vérité - en tant que critère scientifique vérifiable - étant toujours révisable. En fait, ce que perd dans ce raisonnement le philosophe, c'est l'idée que les notions aient un fond, une essence. On pose alors que «les significations ont une identité à travers le temps, mais pas d'essence» (Putnam 1990: 37).

Cette idée de «charité dans l'interprétation» n'est pas très éloignée de ce que Gilles-Gaston Granger appelle le «principe tacite de tolérance sémantique» (1967: 57), principe qui milite en faveur d'une relative imprécision du concept, forme de flou qui facilite son rôle heuristique dans les transferts d'un domaine de connaissance à l'autre.

Nous conclurons de cette brève incursion dans le territoire de la philosophie analytique que :

$\diamond$ le langage découpe une grille dont la pérennité assure l'intercompréhension, quelle que soit la divergence des constructions conceptuelles auxquelles elle sert; 
$\checkmark$ les constructions conceptuelles sont solidaires d'une construction par le récepteur au niveau textuel et discursif;

$\diamond$ l'usage d'une langue naturelle influe nécessairement sur ces constructions conceptuelles;

$\diamond$ l'usage de signes pour impliciter des concepts dépend des communautés d'utilisateurs;

$\diamond$ le statut de concept se trouve attesté par une capacité à agir d'après lui ;

$\diamond$ le concept, en tant que construction individuelle et interactionnelle, doit être distingué de la référence qui renvoie essentiellement à une norme sociale ;

$\diamond$ cette norme est fixée lors du baptême mais seule une similarité relative lie les référés effectifs à la norme.

Ces aperçus permettent de rompre avec un idéalisme gênant pour la réflexion et de restaurer la dimension sociale de la construction dynamique du concept et de la signification.

Toutefois, les arguments qui précèdent tirent une partie de leur force de la coexistence des usages savants et non savants des langues et de l'inclusion des ergolectes dans la langue générale. Or notre réflexion serait invalidée si elle ne prenait en compte l'effort de clôture auquel se livre toute science et dont témoigne la construction d'ensembles conceptuels.

\section{LA FORMALISATION : UN POINT DE VUE DIALECTIQUE}

Cette tension entre un exercice du langage naturel et une formalisation logique close sur elle-même se trouve au cœur de nombreuses réflexions de Noël Mouloud. Son analyse le conduit à envisager la formalisation comme un procédé ouvert :

[...] identifier le rationnel à la clôture, c'est penser dans un contexte exagérément idéaliste: au lieu du concept qui se forme en opérant, on voit un concept dont la compréhension serait en principe circonscrite. (1968:134)

Et c'est, on l'aura compris, vers cette circonscription que tend la pratique terminologique en mettant l'accent sur un seul aspect d'un mouvement que l'auteur nous décrit comme double.

Ce mouvement est celui par lequel la connaissance se rassemble, s'implicite sous le concept et s'extériorise dans les techniques expérimentales. Et c'est dans ce va-et-vient entre effort théorique et mise en pratique que se déploie la praxis scientifique, qui est une «objectivation continuée»: le monde de la connaissance exacte est donc en perpétuel mouvement. C'est pourquoi l'attitude logique s'exclut de cette praxis en considérant des langages totalement explicités. Et coupés de l'histoire.

Et c'est au sein d'un même mouvement historique qu'il convient de distinguer deux usages de la langue que Noël Mouloud oppose comme ceux d'un langage normant, dont la norme est non intentionnelle, et d'un langage auto-normé, qui relève d'une norme constituée à partir des épreuves de la pratique et de la vérification et se distingue ainsi de la norme, non intentionnelle, qui fonde le fait culturel.

Cette opposition, familière aux linguistes, explique la concurrence en langue de valeurs terminologiques, provisoirement exactes, et de stéréotypes, proprement culturels : le stéréotype du chat, à forte charge culturelle, diffère et dépasse ce qu'est le félidé pour lla zoologie.

En fait, la nécessité dans laquelle se trouve toute pensée d'accrocher de la matière autour de formulations abstraites conduit donc à l'inscription des vues de l'esprit dans des modèles métaphoriques, ou au moins imagés (par exemple le modèle de l'atome de Bohr), et à un va-et-vient entre langue naturelle et langages adaptés à une connaissance 
objective. Cette dualité s'inscrit dans le mouvement même de la langue qui, au-delà du mouvement de néologie, contient aussi cette histoire interne, propre à la langue du savoir, qui est faite de significations réinterprétées et de références reprises.

Cette épaisseur sémantique et historique permet de replacer les usages auto-normés des langues scientifiques dans leur relation au fonctionnement général des langues et de dénoncer leur réduction à des codes. Se polariser sur la seule norme volontaire et provisoire, c'est dénier toute sa dimension culturelle à l'activité scientifique et technique.

Du reste, aucun métalangage ne s'en abstrait car, comme le souligne Alain Rey:

[...] même les langages artificiels, qui procèdent par épuration de ce fouillis social, de ce monstre d'ambiguité qu'est le langage humain, n'échappent pas à des conditions sociohistoriques d'élaboration. Il n'en est pas moins vrai que la mathématique, la logique, quelques nomenclatures et terminologies scientifiques, peuvent contourner (en partie) les pièges patiemment montés que recèlent les langues naturelles. (Rey 1990 : 777)

On le voit, il n'est ni possible ni utile d'adopter une position tranchée; mais la polarisation fréquente sur les formalismes et les symbolismes caractéristiques du discours des sciences exactes ne doit pas conduire à oublier qu'il ne s'agit là que d'instruments supplémentaires de précision. De la même façon, si les symboles adhèrent à des trames opératoires dont les calculs sont les formes les plus abstraites, ils fonctionnent comme des termes et se raccordent à une part d'images et de figurations.

On ne saurait donc réduire le pouvoir du langage à l'empire d'un code, au sein duquel la possibilité d'incidences inattendues témoigne de la faculté de novation que seule offre la langue, langue dont tout code reste tributaire.

$\mathrm{Si}$ nous récapitulons en quelques points la conception dialectique de Noël Mouloud, nous en retiendrons que :

$\checkmark$ l'activité scientifique se situe dans un double mouvement entre assignation de valeurs exactes et enrichissement de la langue commune;

$\checkmark \quad$ le concept est lié à une praxis qu'il implicite et qui le vérifie;

$\checkmark$ les liens entre catégories scientifiques et langue naturelle obligent à prendre en compte la dimension historique et culturelle de la science;

$\checkmark \quad$ le recours au langage commun est attesté par le besoin de modèles dont use la pensée scientifique pour «s'incarner» en «s'imageant»;

$\vartheta$ les formalismes et symbolismes ne sauraient autoriser à réduire le langage de la communication scientifique à un code.

\section{LE CONCEPT : LA THÉORIE TERMINOLOGIQUE}

«On peut s'étonner que la terminologie ait tant tardé à préciser sa théorie du concept, car celle-ci fonde toute son activité»: l'étonnement d'Alain Rey (1979: 32) n'a rien perdu de son actualité.

Rappelons rapidement qu'en terminologie, la notion de concept concerne essentiellement le contenu sémantique de certains noms. Mot et terme sont opposés, la signification d'un mot dépendant toujours du contexte dans lequel il est utilisé alors qu'un terme «est affecté à une signification déterminée, c'est-à-dire un concept défini» (Nedobity 1983: 254).

Cette conception est largement partagée. Ainsi, Kocourek délimite la notion de terme par la définition des signes: «le terme est une unité lexicale définie dans les textes de spécialité» (1982: 77). La définition et le concept sont alors tenus pour une seule et même réalité, fixe et indépendante des discours. 


\subsection{L'INDÉTERMINATION DES CONCEPTS}

Partant des problèmes que soulève le rôle capital, en terminologie, des banques de données, Hans Czap en vient à poser que c'est la conception même du concept qui doit être précisée et, de là, il mène une étude critique de la notion de concept telle que l'utilisent les terminologues.

La préoccupation pragmatique de l'auteur le conduit à s'intéresser moins à la notion de concept qu'aux unités d'information qui, selon lui, constituent un concept. Sa conception relève d'une tendance, courante dans le domaine des recherches en intelligence artificielle, selon laquelle connaître, c'est en fin de compte transformer des objets en bits. Or il est intéressant de constater que l'auteur lie étroitement la précision des concepts, requise par l'efficacité des systèmes documentaires, à l'engorgement de ces systèmes. Selon lui :

[...] ce phénomène trouve son origine dans l'indétermination radicale des concepts. Lorsqu'on tente de figer les concepts complexes dans une définition univoque, on évacue forcément un éventail d'autres acceptions. Amputé de la sorte, un concept perd sa référence au réel et partant, son utilité. (Czap 1989:72)

Les concepts sont donc par nature indéterminés et le dilemme auquel est confronté le terminologue, c'est d'être conduit à établir des définitions de concepts si étroites et spécialisées qu'elles finissent en fait par entraver la communication au lieu de la faciliter. On en trouve l'illustration dans la multiplication des homonymes, par exemple.

De fait, la théorie terminologique ne peut répondre aux problèmes que posent les variations minimes de sens qui affectent un concept lors de son passage d'une discipline ou d'un contexte à l'autre, et l'on sait qu'il existe entre les sciences une véritable circulation des concepts qui fait de l'emprunt interdisciplinaire une grande source heuristique. Cela s'explique notamment par le fait que l'acceptation d'une idée se trouve facilitée «lorsqu'elle est rattachée par analogie à un circuit conceptuel déjà frayé» (Schlanger 1971:26).

\subsection{UN PROBLÈME THÉORIQUE ET MÉTHODOLOGIQUE}

La difficulté soulevée ci-dessus est intéressante dans la mesure où elle repose sur le fait que la méthodologie usuelle raisonne sur des ensemblès de termes figés et étrangers les uns aux autres. Le découpage de ces ensembles de termes devant correspondre à des domaines qui se laissent docilement structurer en arbres documentaires. Mais, ce faisant, on oublie que la pensée scientifique et technique ne s'actualise pas sous la forme de «terminosystèmes» donnés à l'avance et que les concepts ne sont pas immuables. C'est pourquoi la persistance dans cette voie nous semble vouée à l'échec et l'on ne peut que regretter que l'institutionnalisation de la pratique terminologique ait précédé le développement d'une réflexion théorique autonome.

C'est sans doute par une démarche plus proche des textes et de la réalité sociale et linguistique que l'on parviendra à mieux décrire ces vocabulaires et les notions qu'ils véhiculent. Il conviendrait peut-être de nuancer le point de vue de Sylvain Auroux, selon qui «les linguistes n'ont jamais vraiment conçu le rapport exact de ces unités aux unités linguistiques, ils se contentent de les exclure de leur champ en les référant à une 'composante encyclopédique' de la compétence linguistique et cognitive» (1990: XI). Mais l'on ne parviendra à mieux appréhender les questions que pose la description des concepts qu'en tenant compte des acquis des sciences du langage contemporaines.

Ce qui précède ne visait qu'à stimuler la réflexion en indiquant des positions et en cernant des problèmes. Mais nous souhaitons, pour ne pas conclure sur une simple revue de questions, exposer la façon, modeste, dont nous avons appréhendé ces difficultés et 
présenter comment nous avons cherché, dans un travail terminographique collectif, à les résoudre, ou à nous y résoudre.

\section{PROPOSITIONS}

Pour aborder ces problèmes, il importe de bien distinguer les deux ordres de faits que désignent les termes de concept et de signifié. Il s'agit moins de les opposer que de les renvoyer à des secteurs particuliers de la réalité. Partant des signes, on pourrait reprendre la terminologie de Hjelmslev et opposer forme et substance du contenu. Les formes du contenu sont du ressort de l'analyse linguistique, les concepts pouvant être appréhendés comme relevant de la substance du contenu.

\subsection{DÉCRIRE LE SENS OU LES CONNAISSANCES?}

Cette distinction est rarement présente dans les travaux ayant pour objet la terminologie, la représentation des connaissances et ces sortes d'applications. On relève d'ailleurs des ambiguïtés dans les formulations mêmes des chercheurs attachés à décrire la signification des termes: la recherche doit-elle se penser comme liée à la valeur linguistique des unités, tâche assignée à la sémantique, ou à un contenu cognitif repérable aux propositions qui le sous-tendent?

Les réseaux associatifs par lesquels on tente de modéliser les connaissances sont fréquemment présentés comme visant à représenter le contenu sémantique ou propositionnel d'unités linguistiques ou de concepts scientifiques (par exemple, Otman 1993). De telles présentations posent question: y a-t-il homologie de traitement de ces deux types de données ou, simplement, indistinction de deux niveaux étroitement intriqués? On retrouve la même ambiguilté quand on insiste sur la double fonction de la relation d'hyperonymie / hyponymie, qui permet à la fois de mettre en rapport des unités lexicales et de construire des classes, des sous-classes et des individus.

Il y a là une méthodologie unitaire qui rend peut-être caduque la distinction signifié / concept, mais qui semble permettre difficilement de décrire la coexistence de valeurs culturelles et de valeurs exactes, et plus encore, la concurrence de valeurs exactes. Cependant, il nous paraît utile de mener notre distinction à son terme, non tant dans le but de poser une séparation arbitraire que dans celui de chercher à mieux comprendre le rapport d'inclusion qui caractérise la relation des terminologies au lexique global d'une langue.

En effet, cette double articulation permet de prendre en compte le fait que les unités conceptuelles sont liées aux réseaux linguistiques, puisque leurs dénominations entrent dans des relations lexicales, ainsi que toute dénomination. L'effort réflexif peut rendre autonome le sens construit par le sujet parlant: c'est un effort de ce type que réclame la construction de la notion physique de masse, en rupture avec le sens habituel. On est alors face à des procès de réassignation sémantique. Mais cette approche lexicaliste ne suffit pas.

\subsection{DES COLLOCATIONS ET DES PARADIGMES}

En effet, les mots sont toujours utilisés dans des énoncés et possèdent leurs collocations, leurs environnements syntaxiques. Cette dimension syntagmatique, qui explique le récent retour d'intérêt pour la phraséologie ( $c f$. Terminologies nouvelles $\mathrm{n}^{\circ} 10$; Terminologie et traduction, 1992, $\mathrm{n}^{\circ} 2$ / 3.), contraint l'usage des signes et, partant, les schèmes qui interviennent dans la construction des concepts correspondants. On trouve une illustration frappante de ce phénomène dans l'une des formes que prend la circulation des concepts, à savoir les emprunts métaphoriques. Par exemple, dans le vocabulaire de la génétique, on parle tout ensemble de transfert d'information, de codage, d'ARN traduit et 
d'ARN messager, de système de lecture, de duplication, de transcription, de cartes physique ou génétique, etc. Il y a là tout un champ métaphorique de l'information et du document dont la cohérence s'applique avec bonheur à un ordre de réalités tout à fait nouveau.

S'il est légitime, nous l'avons vu, de situer le concept à un niveau plus psychologique, il importe de le concevoir comme construit par le biais du langage, plus précisément par la détermination d'un signifié. Par exemple, en parcourant le signifiant chambre à bulles, je puis accéder sans difficulté au signifié, lequel ne me dit presque rien sur le concept. Pour construire le concept, il me faut au moins savoir qu'il s'agit de la dénomination d'un détecteur de particules élémentaires; et ma construction sera bien plus complète si je peux voir ce détecteur et si je le pratique.

Face à champ de vecteurs, la rencontre des signes permet de réduire les possibilités de signifiance des lexies et d'esquisser une notion, d'accéder au moins aux idées d'«espace limité» et de «segment de droite» (voir Thoiron et al. et Boisson, dans ce volume). En revanche, le signifiant ptéridophyte risque fort de constituer un obstacle et fonctionnera aisément comme nom-étiquette, que l'on peut remplacer par fougère, nom du prototype de la classe ainsi désignée. Bien évidemment, dans les cas où la forme linguistique ne circule pas avec un autre usage que purement référentiel, il est bien difficile de mettre en évidence le signifié qui se trouve alors «écrasé». Ce serait le cas également pour charme, nombre quantique, homonyme de charme (arbre) et charme (attrait). C'est dire que l'on se situe bien au niveau d'homonymes appartenant à un paradigme de termes dans lequel se maintient une totale cohérence notionnelle.

Raisonner en termes d'homonymes présente un grand intérêt pour rendre compte de la fonction des signes. La construction de paradigmes dérivationnels permet de distinguer des réseaux associatifs distincts et de mettre en lumière la valeur d'échange des signes. Pour reprendre un exemple cité par Chabridon et Lerat (1993:61), les valeurs d'échange de pénitentiaire et pénitence ne possèdent aucun trait en commun, ils appartiennent à des paradigmes distincts. C'est que la créativité lexicale «s'opère dans une phrase réellement produite, ce qui implique pour le morphème de base un contenu sémantique de terme de discours et non de lexique» (Guilbert 1975: 194).

C'est ainsi que l'apparition, dans la famille dérivationnelle de digérer, du terme digesteur permet de distinguer deux paradigmes, le second paradigme, technique, étant spécialisé pour les procédés permettant la digestion des matières organiques. On peut donc affirmer, sous réserve de tester les limites que l'applicabilité de cette méthode en terminologie, que la pratique des paradigmes lexicaux permet «une analyse de la néologie sémantique en même temps que de la néologie dérivationnelle» (Guilbert 1975: 195).

\subsection{UN EXEMPLE D'APPLICATION}

C'est avec le souci de rester à un niveau de description fonctionnel des unités terminologiques que notre équipe a choisi les rubriques d'analyse de la fiche du dictionnaire des bioindustries Biolex, développé sur logiciel Termex (travail lancé et dirigé, jusqu'à sa disparition, par Louis Guespin, au sein de l'URA CNRS 1164, à l'Université de Rouen). Ces rubriques sont de deux types.

Les premières, lexicales, comprennent, outre celles de synonymie et d'antonymie, les relations d'hyperonymie, d'hyponymie et d'isonymie. On trouve parfois les isonymes dénommés co-hyponymes, notre choix visant seulement à souligner que deux unités de même niveau hiérarchiques pouvaient s'opposer l'une l'autre sans être dominées par une unité d'extension plus large, la notion de co-hyponymie, quant à elle, impliquant celle d'hyperonymie. C'est ainsi qu'autobus et autocar sont isonymes sans être dominés par un hyperonyme direct ( $c f$. Assal et alii 1992). Dans notre corpus, les termes néphélométrie et 
turbidimétrie sont isonymes et ne pourraient être englobés que par une paraphrase du type «technique de mesure de la turbidité», pas par une unité lexicale attestée.

Les rubriques suivantes, prédicatives, sont inspirées des fiches proposées par Pierre Lerat pour les travaux du Centre de terminologie et de néologie, dont il a été le fondateur. L'idée initiale était d'explorer le rendement des propriétés de

prédicat privilégié («action typique») et d'argument logique contigu («objet connexe») dans une relation prédicative de premier ordre. Dans la rubrique «objet», il s'agit de repérer les notions qui sont associées le plus spontanément par les experts (la cause, le prévenu, le tribunal, autour de l'action «procès», pour le juge, par exemple). (Lerat 1988:18)

C'était là permettre d'enregistrer des faits syntagmatiques significatifs et non plus seulement des faits paradigmatiques. Et cette direction rencontrait notre souci de concevoir un dictionnaire qui évite la démarche métalinguistique consistant à rédiger des définitions. Pour ce faire, notre pari était de recenser suffisamment de contextes pour permettre au lecteur de construire le concept correspondant, avec une richesse de points de vue que ne pourrait proposer aucune rédaction de définition. Cependant, nous souhaitions extraire de nos contextes des renseignements syntagmatiques concis susceptibles de fournir matière à des rubriques.

Durant le cours de ce travail, nous avons été conduits à inclure quatre rubriques. Celles d'«action typique» et d'«objet typique» ont été complétées par les rubriques d'«agent typique» et d'«application typique».

Prenons comme exemple la description du terme filtre bactérien.

La rubrique $\{$ act.typ $\}$ permet d'indiquer qu'un filtre bactérien sert le plus souvent (c'est là le sens de typique) pour l'épuration.

La rubrique \{obj.typ\} permet d'indiquer l'objet typique de l'action concernée, ici qu'un filtre bactérien sert le plus souvent pour les effluents urbains.

La rubrique \{ag.typ\} concerne ce qui fait le plus souvent l'action, dans le cas du filtre bactérien, l'agent typique ce sont les micro-organismes.

Enfin, la rubrique \{app.typ\} permet de repérer les applications, les procédés dans lesquels les actions sont le plus fréquemment utilisés. Cette rubrique permet de repérer des renseignements du type «domaine», mais à un niveau de finesse variable selon les nécessités. Par exemple, le contrôle organoleptique a pour application typique l'industrie alimentaire, cela concerne toute la filière; mais le clonage moléculaire a pour application typique l'obtention de sondes d'ADN. Pour reprendre filtre bactérien, son application typique est la dépollution.

Une fois ces renseignements extraits de nos sources documentaires, nous pouvons construire une sorte de phrase de base élémentaire et dire que le filtre bactérien est utilisé pour l'épuration des effluents urbains au moyen de micro-organismes dans la dépollution. Il s'agit là d'une méthode d'analyse permettant de donner des énoncés typiques, linguistiquement représentatifs des unités décrites.

Les quatre rubriques que nous avons utilisées ne sont d'ailleurs pas limitatives; d'autres existent. Songeons aux propositions de la norme ISO 1087, qui mentionne les relations pragmatiques et séquentielles, incluant par exemple, les relations cause-effet, producteur-produit ou étapes d'un processus (ISO 1990: 3). Par ailleurs, des descriptions de vocabulaires différents pourraient faire progresser la recherche et mettre en lumière la nécessité de nouvelles rubriques. C'est ainsi que nous avons été conduits à ajouter à notre fiche la rubrique «application typique» à cause de notre volonté d'éviter les notions de type documentaire, comme celles de domaine, sous-domaine, pour chercher à décrire plus précisément les conditions socioprofessionnelles d'usage des termes. En cela, nous 
retrouvions les insatisfactions qui avaient conduit certains terminographes, comme Gabriel Otman, à utiliser des rubriques plus fines, comme celles de domaine d'application.

Nous avons pris un exemple relativement simple, qui permet de cerner rapidement l'économie d'une telle méthode d'analyse. Il existe bien sûr des difficultés dans l'application d'une telle méthode, mais l'ensemble du travail accompli permet de dire qu'une telle approche linguistique de tels vocabulaires est tenable. Les connaissances accumulées sur les énoncés métalinguistiques, sur les reformulations permettent de limiter les risques d'erreurs dans la consignation des renseignements: il s'avère que l'on repère avec assez de précision des contextes définitoires.

Par ailleurs, l'analyse en rubriques et l'évitement d'énoncés définitoires et définitifs permet de proposer une restitution en termes de typicité des renseignements présents dans les discours. Ces discours n'étant pas restituables, leur analyse en termes d'actions, objets, agents et applications typiques permet d'en proposer une modélisation faible, une sorte de moyenne.

Et c'est ici que l'on remarque l'intérêt qu'il y a, au plan de la méthode, à réduire dans un premier temps les concepts à un ensemble de discours dont le terminographe ne peut que restituer des linéaments. La question que le lecteur ne peut manquer de soulever est celle de savoir dans quelle mesure on ne retrouve pas la simple définition, après un détour bien tortueux...

Nous illustrerons l'opposition que nous cherchons à esquisser par notre corpus. Le signifié de fermentation est assez simple à décrire, il s'agit d'un nom d'action, qui s'oppose par exemple à agitation, ébullition, et dont le paradigme dérivationnel comprend fermenter, fermenteur, fermentaire, fermentatif... Le concept traditionnel de fermentation comportait le trait caractéristique «en absence d'oxygène» (fermentation anaérobie). Mais, dans le cadre des applications bioindustrielles, ont été développées des fermentations industrielles en présence d'oxygène (fermentations aérobies). ll en est résulté une ambiguïté possible pour le terme fermentation qui correspond à deux concepts différents, l'un, traditionnel, anaérobie et l'autre, récent, aérobie.

Autre exemple, température de fusion possède un signifié simple, comme beaucoup de dénominations analytiques, or notre dictionnaire décrit, pour ce même signifié, deux concepts différents selon que les objets typiques sont les brins d'une molécule ou des matières grasses, les actions étant dans un cas la séparation des brins, dans l'autre le changement d'état solide-liquide.

\section{COMMENT DÉCRIRE LA PLASTICITÉ DU SENS?}

L'approche en rubriques est bien évidemment réductrice, mais nous pensons que cet appauvrissement par l'analyse est compensé, dans une telle méthode, par la mise à disposition de contextes authentiques. Ces contextes possèdent selon nous deux vertus. D'une part, quand ils livrent des renseignements comparables, ils entrent dans un espace de reformulations et l'on sait que les reformulations aident à l'appropriation des concepts. D'autre part, les contextes offrent fréquemment sur l'objet des points de vue différents, mais aussi légitimes et vrais, auquel cas la diversité des discours recueillis autorise une élaboration cognitive plus riche. Aussi la conjugaison des deux facteurs nous semble constituer, au-delà de la seule construction du sens, une aide pour l'élaboration des connaissances.

Par ailleurs, en choisissant un tel mode descriptif, on restitue un peu de la «plasticité» des concepts, dont on sait qu'ils ne fonctionnent que rarement comme des catégories fixes et closes. D'ailleurs, cette intuition de linguistes rejoint les données 
récentes de la neurologie. En effet, comme l'affirme Steven Rose, les concepts ne sauraient être pris pour des ensembles figés de données, car:

[...] le cerveau ne travaille pas sur de l'information, mais sur du sens. Et le sens est quelque chose qui est façonné par l'histoire et le développement, et est exprimé par les individus en interaction avec leur environnement social et naturel. (Rose 1994 : 115)

On ne peut donc pas considérer que l'on va chercher les concepts dans leur lieu de stockage et qu'une fois consultés, on les y remet, non modifiés. Bien au contraire, l'acquisition des concepts est plutôt une appropriation, qui suppose une acclimatation et une «négociation cognitive», la construction de nouvelles catégories supposant une réorganisation des catégories antérieures.

Il importe donc de garder présent à l'esprit que les unités correspondant à des concepts sont labiles; elles se rattachent à des discours habituels mais variables, ce que les notions d'extension et d'intension condamnent à négliger. Leurs reprises, leurs réutilisations ne se font pas dans la fixité livresque de définitions, exception faite des concepts formalisables. Leurs validations initiales ne les abstraient pas ensuite des réseaux langagiers et cognitifs ultérieurs dans lesquels ils se trouvent pris, repris, modifiés et enrichis. Car si la souplesse, la marge inhérente de jeu et l'évolutivité sont des qualités que les linguistes s'accordent à faire figurer parmi les conditions des interactions langagières, on peut légitimement croire qu'elles figurent également parmi celles les conditions nécessaires à l'activité cognitive. Et de ce point de vue, l'approche sémantique de la vulgarisation scientifique constitue un terrain très riche d'enseignements.

Note

1. L'auteur remercie de sa lecture attentitve Anne Condamines, URA CNRS 1033, Université ToulouseLe Mirail.

\section{RÉFÉRENCES}

APEL, K.O. (1987): L'éthique à l'âge de la science, Lille, Presses Universitaires de Lille, 140 p.

ASSAL, Allal et alii (1992): «Sémantique et terminologie: sens et contextes», Terminologie et traduction, $\mathrm{n}^{\circ} 2 / 3$, Commission des communautés européennes, pp. 411-421.

AUROUX, Sylvain (1990): «Avant-propos», Encyclopédie philosophique universelle. T. II. Les notions, Paris, PUF, pp. VII-XX.

CASSIRER, Ernst (1972): La philosophie des formes symboliques. T. 3. La phénoménologie de la connaissance, Paris, Minuit, $616 \mathrm{p}$.

CASSIRER, Enst (1977): Substance et forction. Éléments pour une théorie du concept, Paris, Minuit, $429 \mathrm{p}$.

CHABRIDON, Jacky et Pierre LERAT (1993): «Terme et famille de termes», La banque des mots, numéro spécial, CILF, pp. 55-63.

COSSUTTA, Frédéric (1989): Éléments pour la lecture des textes philosophiques, Paris, Bordas, 244 p.

CZAP, Hans (1989): «Le concept de concept», Terminologie diachronique, éd. CILF et Communauté française de Belgique, pp. 69-74.

GAUDIN, François (1993a) : «Socioterminologie: du signe au sens, construction d'un champ», Meta, 38 (2), Presses de l'Université de Montréal, pp. 293-301.

GAUDIN, François (1993b): «Le praxème: un modèle pour une théorie du terme?», Langage et praxis, Montpellier, Praxiling, pp. 68-76.

GAUDIN, François (1993c): «Champs, clôtures et domaines: des langues de spécialités à la culture scientifique», Les langues de spécialité : pratiques, outils, théories. Actes de la 4ème journée ERLA-GLAT, Brest, éd. E.N.S. Télécom Bretagne, pp. 25-40.

GAUDIN, François (1993d): Pour une socioterminologie: des problèmes sémantiques aux pratiques institutionnelles, Publications de l'Université de Rouen, $254 \mathrm{p}$.

GOUPIL, Michelle (1988): «Transferts mutuels de vocabulaire: psychophysiologie et physicochimie», Transferts de vocabulaire dans les sciences, éd. CNRS, pp. 125-133.

GRANGER, Gilles-Gaston (1967): Pensée formelle et sciences de l'homme, Aubier-Montaigne, $226 \mathrm{p}$.

GUILBERT, Louis (1975): La créativité lexicale, Paris, Larousse, 285 p.

ISO (1990): Terminologie. Vocabulaire, Norme internationale ISO 1087. 
KOCOUREK, Rostislav (1982): La langue française de la technique et de la science, Wiesbaden, Brandstetter, $259 \mathrm{p}$.

KUHN, Thomas S. (1972) : La structure des révolutions scientifiques, Paris, Flammarion, 246 p.

KRIPKE, Saül (1982) : La logique des noms propres, Paris, Minuit, $173 \mathrm{p}$.

LAFONT, Robert (1993): Le dire et le faire, Montpellier, Praxiling, 349 p.

LERAT, Pierre (1988): «Terminologie et sémantique descriptive», La banque des mots, $\mathrm{n}^{\circ}$ spécial, Paris, CILF, pp. $11-30$.

MAURO, Tullio de (dir) (1985): Cours de linguistique générale, de Saussure, édition critique, Paris, Payot, $520 \mathrm{p}$.

MOULOUD, Noël (1968): Les structures, la recherche et le savoir, Paris, Payot, $307 \mathrm{p}$

MOULOUD, Noël (1976) : L'analyse et le sens. Essai sur les préalables sémantiques de la logique et de l'épistémologie, Paris, Payot, $335 \mathrm{p}$.

MORTUREUX, Marie-Françoise (1984): «La dénomination: approche socio-linguistique», Langages, $\mathrm{n}^{\circ} 76$, pp. 95-111.

NEDOBITY, Wolfgang (1983): «La terminologie et son application à la classification, à l'indexation et à l'analyse», RUSIBA, vol. V, $\mathbf{n}^{\circ} 4$, Presses de l'UNESCO, pp. 248-256.

OTMAN, Gabriel (1993): «La représentation de la relation d'hyperonymie / hyponymie», ICO Québec, hiver 1993, pp. 4-11.

PUTNAM, Hilary (1984): Raison, histoire et vérité, Paris, Minuit, $242 \mathrm{p}$.

PUTNAM, Hilary (1990): Représentation et réalité, Paris, Gallimard, coll. «NRF Essais», 226 p.

PUTNAM, Hilary (1992): Définitions. Pourquoi ne peut-on naturaliser la raison?, éd. de l'Éclat, $96 \mathrm{p}$

REY, Alain (1977): Le lexique : images et modèle. Du dictionnaire à la lexicologie, Paris, Armand Colin, $307 \mathrm{p}$.

REY, Alain (1979): La terminologie, noms et notions, Paris, PUF, coll. «Que sais-je?», $\mathrm{n}^{\circ} 1780,127 \mathrm{p}$.

REY, Alain (1990): «Lexico-logiques, discours, lexiques et terminologies 'philosophiques'», Encyclopédie philosophique universelle. T. II. Les notions, Paris, PUF, pp. 775-781.

ROSE, Steven (1994): La mémoire. Des molécules à l'esprit, Paris, Le Seuil, 403 p.

SAUSSURE, Ferdinand de (1985): Cours de linguistique générale, édition critique de Tullio de Mauro, Paris, Payot, $520 \mathrm{p}$.

SCHLANGER, Judith (1971): Les métaphores de l'organisme, Paris, Libraire J. Vrin, 269 p.

SLODZIAN, Monique (1993): «La V.G.T.T. (Vienna General Theory of terminology) et la Conception Scientifique du Monde», Le langage et l' homme, vol. XXVIII-4, pp. 223-232.

WITTGENSTEIN, Ludwig (1961): Investigations philosophiques, Paris, Gailimard, 368 p. 